The role of free electrons in the guiding of positive streamers

This content has been downloaded from IOPscience. Please scroll down to see the full text.

2016 Plasma Sources Sci. Technol. 25044001

(http://iopscience.iop.org/0963-0252/25/4/044001)

View the table of contents for this issue, or go to the journal homepage for more

Download details:

IP Address: 192.16.191.140

This content was downloaded on 30/03/2017 at 13:49

Please note that terms and conditions apply.

You may also be interested in:

Streamer discharges can move perpendicularly to the electric field

S Nijdam, E Takahashi, J Teunissen et al.

Investigation of positive streamers by double-pulse experiments, effects of repetition rate and gas mixture

S Nijdam, E Takahashi, A H Markosyan et al.

Nanosecond repetitively pulsed discharges in N2-O2 mixtures: inception cloud and streamer emergence She Chen, L C J Heijmans, Rong Zeng et al.

Probing photo-ionization: simulations of positive streamers in varying N2:O2-mixtures

G Wormeester, S Pancheshnyi, A Luque et al.

Experiments on streamers in pure gasses and mixtures

S Nijdam, F M J H van de Wetering, R Blanc et al.

Probing background ionization: positive streamers with varying pulse repetition rate and with a radioactive admixture S Nijdam, G Wormeester, E M van Veldhuizen et al.

3D PIC-MCC simulations of discharge inception around a sharp anode in nitrogen/oxygen mixtures Jannis Teunissen and Ute Ebert

Pulsed positive discharges in air at moderate pressures near a dielectric rod

A Dubinova, D Trienekens, U Ebert et al.

Streamer propagation in a weak uniform field

Yu V Serdyuk, A Larsson, S M Gubanski et al. 


\title{
The role of free electrons in the guiding of positive streamers
}

\author{
S Nijdam ${ }^{1}$, J Teunissen ${ }^{2}$, E Takahashi ${ }^{3}$ and U Ebert ${ }^{1,2}$ \\ ${ }^{1}$ Department of Applied Physics, Eindhoven University of Technology, PO Box 513, \\ 5600 MB Eindhoven, The Netherlands \\ 2 Centrum Wiskunde \& Informatica (CWI), Amsterdam, The Netherlands \\ 3 National Institute of Advanced Industrial Science and Technology (AIST), 1-2-1 Namiki, \\ Tsukuba Ibaraki 305-8564, Japan \\ E-mail: s.nijdam@tue.nl
}

Received 29 February 2016, revised 8 April 2016

Accepted for publication 22 April 2016

Published 27 May 2016

\begin{abstract}
Because positive streamers propagate opposite to the electron drift velocity, their growth not only depends on the local electric field, but also on the electron density ahead of them. We have recently demonstrated the importance of this electron density, by showing that positive streamers could be guided by weak preionization from a laser. Here, we study streamer guiding in nitrogen-oxygen mixtures in more detail, to better understand the propagation of positive streamers. First, the conditions required for guiding are investigated, both experimentally and with three-dimensional numerical simulations. Particular focus is on the role of oxygen and photoionization, which tend to inhibit guiding. Then the position of the guided streamers is investigated, and successfully related to the electron drift.
\end{abstract}

Keywords: streamer, plasma, discharge, laser guiding, pure nitrogen, 3D simulations

(Some figures may appear in colour only in the online journal)

\section{Introduction}

Streamers are rapidly growing ionized channels that enhance the electric field at their tips [1]. They are the first stage of sparks and they are relevant for both industrial and medical processes [2-6] as well as for geophysical phenomena like sprites and lightning [7-9]. Streamers can occur in a variety of gases [10] as well as in liquids [11].

A positive streamer generally propagates along the direction of its 'background' electric field, i.e. the combined field of the electrode configuration and neighboring discharges [12]. However, we have recently shown that a modest local increase in the background ionization, caused by laser illumination, can cause streamers to strongly deviate from this direction. In [13], we studied the laser guiding of streamers in nitrogen-oxygen mixtures at 133 mbar, using $4-10 \mathrm{kV}$ positive pulses, a $103 \mathrm{~mm}$ point-plane geometry and a laser preionization level of about $10^{9} \mathrm{~cm}^{-3}$. Under these conditions, guiding only occurred for oxygen concentrations below $0.5 \%$. In [14] we showed that guiding also occurs in pure argon.
Note that the laser guiding mechanism used here is quite different from other laser guiding experiments in literature. Other experiments generally operate in ambient air where a focused high intensity laser beam creates a highly conductive path with a high ionization degree [15-18]. This is in contrast with our experiments where a low intensity laser beam creates a modest ionization degree in nitrogen with small oxygen admixtures.

Now, we study streamer guiding in more detail in order to better understand the propagation of positive streamers. Because such streamers propagate opposite to the electron drift velocity, they require a source of free electrons in front of them [10, 19, 20]. Our previous studies on laser guiding [13] and complex pulse shapes [21, 22] have already shown that remaining background ionization can have a great influence on streamer propagation direction.

In the laser guiding study [13] it was found that laser guiding does not occur for air and other nitrogen-oxygen mixtures with high oxygen concentrations. In section 3 , we show that the reason is probably the photoionization [10, 23, 24] of oxygen molecules. In nitrogen-oxygen mixtures, this 


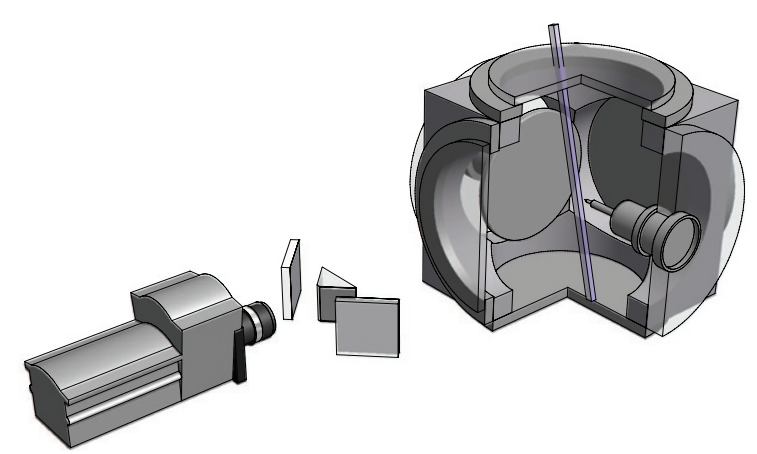

Figure 1. Schematic overview of the set-up including, from left to right, the ICCD camera, stereoscopic set-up and vacuum vessel with indication of laser beam path. In the presented images the recorded images are rotated anti-clockwise by $90^{\circ}$ so that the electrode tip is shown on top. Image reproduced with permission from [13].

process can create relatively high electron densities in front of a propagating streamer. A competing effect in oxygen-rich mixtures is the loss of free electrons due to attachment to (electro-negative) oxygen molecules [25].

In [13], the guided streamers were often displaced relative to the preionization trail. In section 4 , we show that this can be explained by electron drift in the background field.

\section{Set-up and methods}

\subsection{Laser guiding experiments}

The experiments treated in this work have been performed with the same set-up that was used and described in [13], which is also depicted in figure 1. This set-up consists of a vacuum vessel, a camera with stereoscopic optics and a $\mathrm{KrF}$ laser.

The vacuum vessel contains a point-plane electrode geometry with a $103 \mathrm{~mm}$ separation, and it is filled with various gas mixtures at pressures between 25 and 250 mbar. The gas mixtures used are pure nitrogen, artificial air $\left(80 \% \mathrm{~N}_{2}, 20 \% \mathrm{O}_{2}\right)$ and other nitrogen oxygen-mixtures. The estimated level of impurities (mostly water and air) in all gas mixtures is about $10 \mathrm{ppm}$.

A laser beam from the $\mathrm{KrF}$ excimer laser enters the vacuum vessel through a large quartz window. The laser produces pulses of 1-2 mJ per pulse at a wavelength of $248 \mathrm{~nm}$ and a pulse length of $20 \mathrm{~ns}$. The (unfocused) laser beam is shaped by four shutters to a quasi-rectangular beam with a height of $1-17 \mathrm{~mm}$ and a width (perpendicular to the image plane as defined below) of 1-12 $\mathrm{mm}$. In all measurements presented here the laser beam crosses the symmetry axis of the vessel and is perpendicular to the viewing direction. The laser pulse will locally increase the ionization level in the gas. In section 2.2 we describe measurements of the laser-induced ionization density and give estimates for this density.

A high voltage pulse forming circuit is used to create a quasi-rectangular positive voltage pulse on the pointed electrode tip some nano- to milliseconds after the laser pulse. These voltage pulses have lengths of 600-1500 ns, rise/fall times of about $15 \mathrm{~ns}$ and amplitudes between 3 and $10 \mathrm{kV}$. The vacuum vessel and pulse forming network are described in more detail in [21].
The high voltage pulses create a streamer discharge in the vessel, which is imaged by an Andor ICCD-camera with stereoscopic optics between it and the vessel [12, 13, 26]. Like in [13], the angle between the two image paths is $24-28^{\circ}$ and the orientation is such that streamers moving perpendicular to the symmetry axis of the point-plane geometry can be properly imaged. The streamer images shown here are presented as anaglyphs with red and cyan colours for images from the two respective cameras like discussed in [13]. An example of such an image is given in figure 2(a). From such measurements we have reconstructed the location of many straight streamer path sections. The 3D locations of some of these streamer sections are plotted in figures 2(c) and (d) and the location of clearly guided sections with respect to the laser beam is plotted in figure 2(b). In this example only the four points from the measurement from figure 2(a) are plotted but in each of the following plots of this type, data of about ten discharges with two to four points per discharge is collected.

\subsection{Laser induced ionization density measurement}

The laser-induced ionization density has been determined in the same vessel as used for the streamer guiding experiments. For these measurements the point-plane geometry is replaced by a plane-plane geometry with a separation of $30 \mathrm{~mm}$ as is shown in figure 3(a). One of the two electrodes is grounded while a fixed DC potential between 0 and $2000 \mathrm{~V}$ is applied to the other electrode. The laser beam is located exactly between the two electrodes. The grounded electrode contains a small sampling electrode with a diameter of $3 \mathrm{~mm}$ in its centre. We assume that the charge drifts in the applied electric field without changing it. The current through the sampling electrode after a laser pulse is now a measure for the ionization density induced by the pulse. In figure 3(b) an example of the voltage over a $1 \mathrm{M} \Omega$ shunt resistor connected to the sampling electrode is shown as function of time after the laser pulse. It contains a slow and a fast component. The fast component is likely caused by the electrons and simple ions (e.g. $\mathrm{N}_{2}^{+}$and $\mathrm{N}_{4}^{+}$) while the slow component may be due to larger water clusters due to impurities. The measured current is integrated and then halved to account for positive and negative charges. This gives an estimate for the number of ionizations in the volume delimited by the laser beam and the area of the sampling electrode (in this case $\pi(1.5)^{2} \times 10 \mathrm{~mm}^{3}$ ).

For each gas mixture and pressure this procedure is performed as function of applied voltage. An example of such a measurement is plotted in figure 4 . In this figure the measured electron density saturates above an applied voltage of about $500 \mathrm{~V}$. Below this voltage the measured ionization density is probably lower due to recombination during the longer drift time. At voltages above $2 \mathrm{kV}$ an increase in measured density is expected due to secondary ionization.

In figure 5 the measured laser-induced ionization density is plotted as function of the laser power density. It is found that the ionization density scales with the laser power density to the power 1.66. This indicates that the ionization mechanism is probably a mixture of single-photon and two-photon 

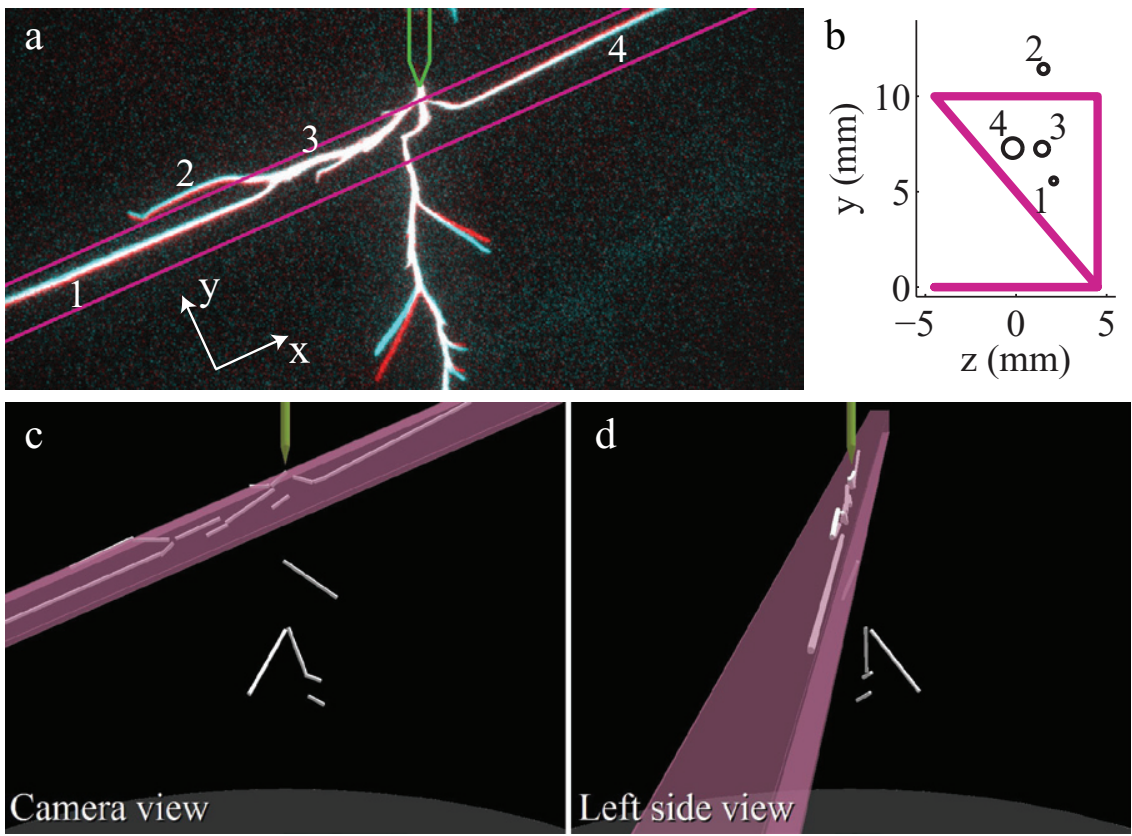

Figure 2. (a) Example of stereo-photography image with the laser trail indicated by the purple lines and the anode tip indicated in green. Here the laser trail is oriented under a non-perpendicular angle with the electrode axis, like in figure 1. Measured parts of the streamer trails are indicated by numbers. (b) Plot of the position of the numbered streamer trails with respect to the location of the laser beam indicated as the purple rectangle. Here the $z$-coordinate is perpendicular to the plane of view. The size of the markers is proportional with the $x$ coordinate with the origin at the left side of the image. (c) and (d) 3D reconstruction of the streamer discharge from (a) like observed from the camera viewpoint (c) and rotated $85^{\circ}$ to the right around the electrode axis (d). Note that the parts of the channel that are nearly vertical in (a) cannot be reconstructed due to the limitations of the stereo-photography method.
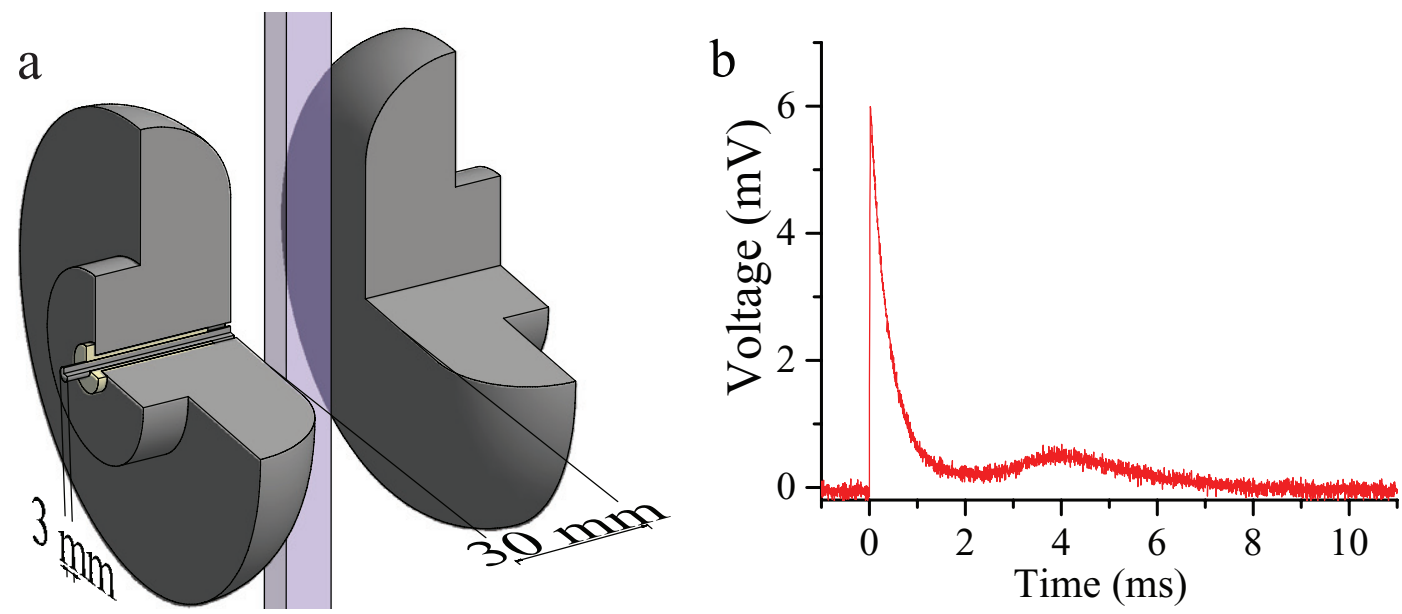

Figure 3. (a) Schematic representation of the set-up used to measure the ionization density and (b) example of a typical measurement of the voltage over the $1 \mathrm{M} \Omega$ shunt resistor connected to the sampling electrode. The laser pulse is applied at $t=0$. Gas: $133 \mathrm{mbar}$ nitrogen, voltage: $1 \mathrm{kV}$.

processes but the exact process is outside the scope of this paper. We can conclude that the laser-induced ionization densities are in the order of $10^{8}-10^{9} \mathrm{~cm}^{-3}$ for the highest laser power, which we used in the experiments.

\subsection{Simulation model and conditions}

In [13] we used a 3D particle model to simulate streamer guiding, but such simulations are computationally quite costly. In order to simulate the larger systems for longer times, we here switch to a 3D plasma fluid model, using adaptive mesh refinement and OpenMP parallelism (see [27], chapter 10). The model is of the drift-diffusion-reaction type:

$$
\begin{gathered}
\partial_{t} n_{\mathrm{e}}=-\nabla \cdot \vec{j}_{\mathrm{e}}+\bar{\alpha}\left|\vec{j}_{\mathrm{e}}\right|+S_{\mathrm{ph}}, \\
\partial_{t} n_{\mathrm{i}}=\bar{\alpha}\left|\vec{j}_{\mathrm{e}}\right|+S_{\mathrm{ph}} \\
\vec{j}_{\mathrm{e}}=-\mu_{\mathrm{e}} n_{\mathrm{e}} \vec{E}-D_{\mathrm{e}} \nabla n_{\mathrm{e}}
\end{gathered}
$$

where $n_{\mathrm{e}}$ is the electron density, $n_{\mathrm{i}}$ the positive ion density, $\overrightarrow{j_{\mathrm{e}}}$ the electron flux, $\vec{E}$ the electric field and $S_{\mathrm{ph}}$ a source term 


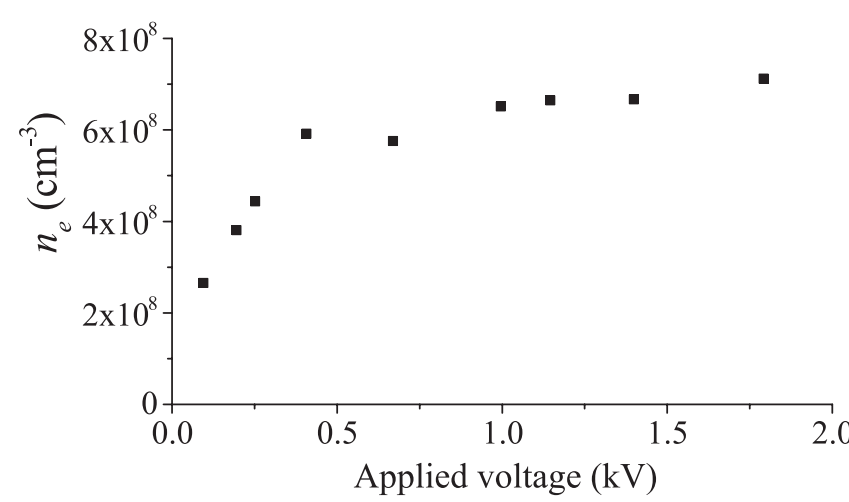

Figure 4. Measured ionization density as function of applied voltage in 133 mbar pure nitrogen.

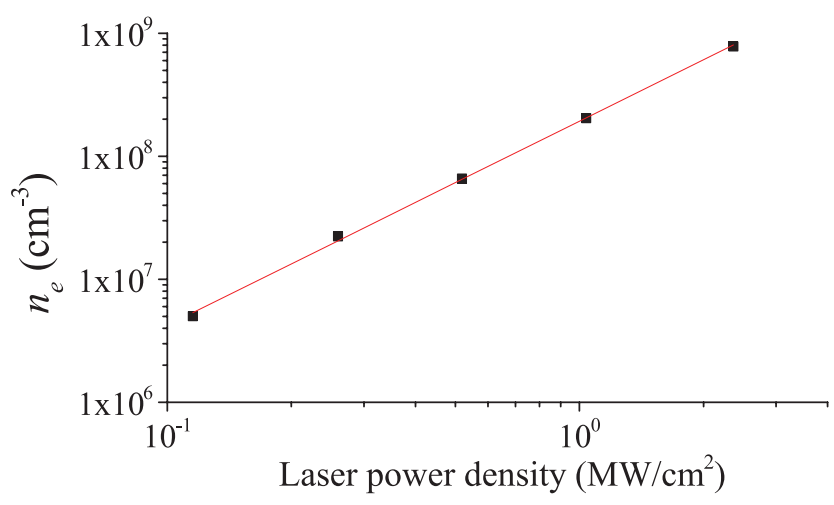

Figure 5. Measured ionization density as function of laser power density. The red line is a linear fit of the points on the double log scale. It has a slope of 1.66 .

due to photoionization. We use the local field approximation, in which the coefficients $\bar{\alpha}$ (ionization minus attachment), $\mu_{\mathrm{e}}$ (mobility) and $D_{\mathrm{e}}$ (diffusion) depend on the local electric field strength $|\vec{E}|$. The electric field is computed in the electrostatic approximation:

$$
\begin{gathered}
\vec{E}=-\nabla \phi, \\
\nabla^{2} \phi=-e\left(n_{\mathrm{i}}-n_{\mathrm{e}}\right) / \varepsilon_{0},
\end{gathered}
$$

where $\phi$ is the electric potential, $\varepsilon_{0}$ the permittivity of vacuum and $e$ the elementary charge. Poisson's equation is solved with the geometric multigrid routines described in chapter 10 of [27], which also describes the explicit time stepping and the numerical flux calculations.

The simulations are performed in $\mathrm{N}_{2}$ containing $0,0.1 \%$ and $1 \% \mathrm{O}_{2}$, at 133 mbar. In all cases, a background ionization level of $n_{\mathrm{e}}=n_{\mathrm{i}}=1.5 \times 10^{5} \mathrm{~cm}^{-3}$ is included. This was approximately the lowest background ionization level at which we could reasonably simulate a streamer in pure nitrogen, i.e. without photoionization. To focus on the differences caused by photoionization, we use $\mathrm{N}_{2}$ transport coefficients (e.g. $\left.\mu_{\mathrm{e}}, \alpha\right)$ for all mixtures, so that electron attachment and detachment reactions are not included. The transport coefficients were computed from the Siglo cross sections of [28], using Bolsig + [29]. Photoionization is computed according to Zheleznyak's model [30], but using a Monte Carlo method with discrete photons (see [31] and chapter 11

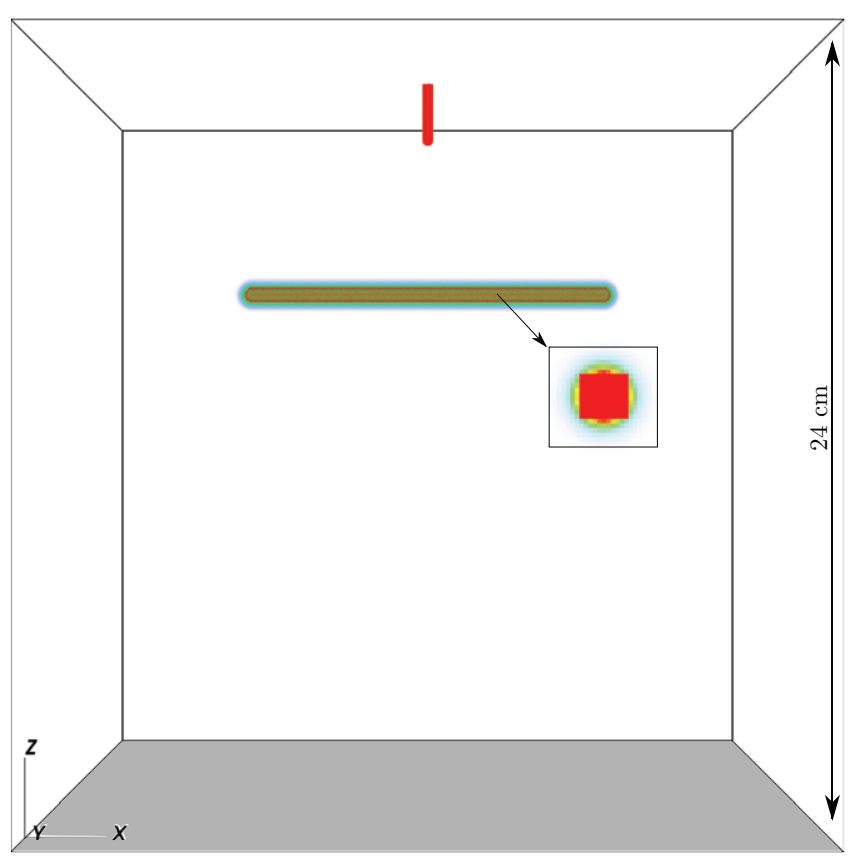

Figure 6. A 3D view of the computational domain, showing the position of the 'laser' ionization (horizontal) and the initial seed from which the streamer starts (top, center). The grounded bottom of the domain is colored gray, and the inset shows a zoomed cross section of the laser ionization.

of [27]). In this model, the absorption function of ionizing photons is given by

$$
f(r)=\frac{\exp \left(-\chi_{\min } p_{\mathrm{O}_{2}} r\right)-\exp \left(-\chi_{\max } p_{\mathrm{O}_{2}} r\right)}{r \ln \left(\chi_{\max } / \chi_{\min }\right)},
$$

where $\chi_{\max } \approx 1.5 \times 10^{2} /(\mathrm{mm} \mathrm{bar}), \chi_{\min } \approx 2.6 /(\mathrm{mm} \mathrm{bar})$ and $p_{\mathrm{O}_{2}}$ is the partial pressure of oxygen. The number of photons produced in a cell during a time $\Delta t$ is determined using random numbers, and the mean number is given by

$$
N_{\gamma}=\eta \frac{p_{q}}{p_{q}+p} N_{\mathrm{I}}
$$

where $N_{\mathrm{I}}$ is the number of ionizations that have taken place, and $p$ is the pressure. As a quenching pressure we take $p_{q}=40$ mbar and we set the constant of proportionality $\eta$ to a field-independent value of 0.05 . At every time step (about 10 ps) up to $4 \times 10^{5}$ 'physical' photons can be produced, above that 'super-photons' are used. These photons are assumed to be isotropically distributed, and their absorption length is sampled from equation (6). In pure nitrogen, photoionization is disabled.

The computational domain of $(24 \mathrm{~cm})^{3}$ is shown in figure 6 . On the top and bottom, a fixed potential is applied to generate a background field of $2 \mathrm{kV} \mathrm{cm}^{-1}$, whereas Neumann zero (e.g. $\partial_{x} \phi=0$ ) boundary conditions are applied on the four other sides. Neumann zero boundary conditions are also used for the species densities, on all sides. We have not yet implemented curved electrodes in our Poisson solver, so we instead place an ionized seed at the top of the domain to generate an initial streamer. This seed has a density of $7.5 \times 10^{12} \mathrm{~cm}^{-3}$ electrons and ions, a height of about $2 \mathrm{~cm}$ and a width of about $3.5 \mathrm{~mm}$, 


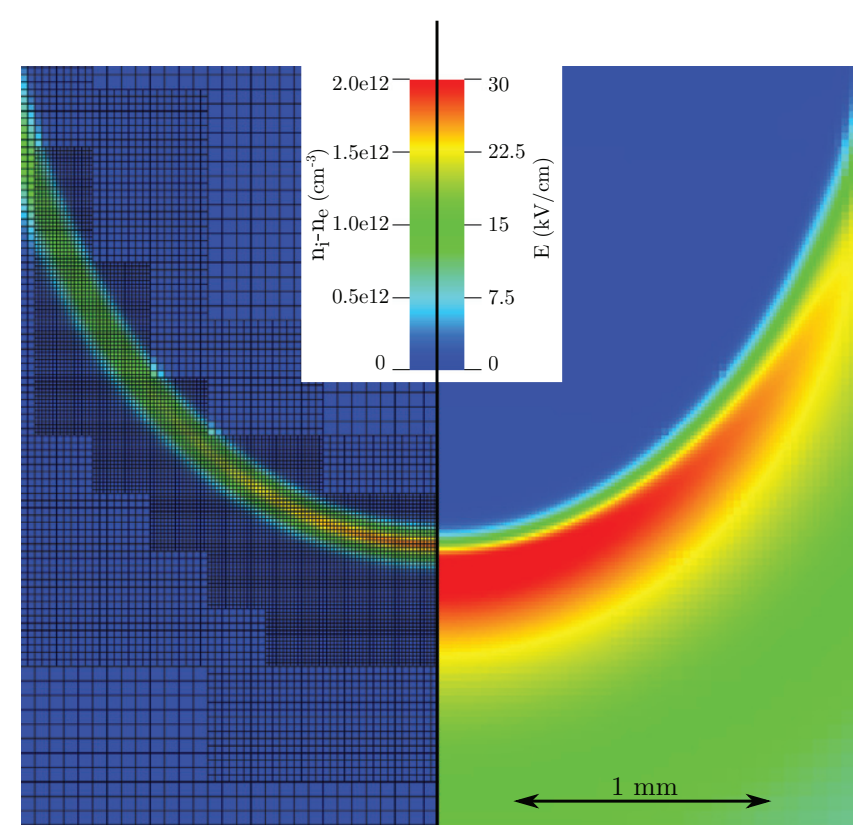

Figure 7. Cross section of a streamer head, corresponding to the case of figure 8(b) at $t=50 \mathrm{~ns}$, see also figure 9. On the left the net charge density is shown together with the numerical mesh, the electric field strength is shown on the right.

using a so-called smoothstep function for the density profile. The electric field at the tip of the seed quickly increases and after a short delay a thin streamer develops. This delay is quite sensitive to the seed's properties, but the further streamer evolution is not. The 'laser' ionization is placed $7.2 \mathrm{~cm}$ below the top of the domain, between $x=8$ and $16 \mathrm{~cm}$ and centered in the $y$-direction. The channel has a cross section of about $(5 \mathrm{~mm})^{2}$, as shown in figure 6 .

There is an important difference between the background field in the computational domain and the background field in the experimental set-up: in the experiments the sides of the vessel are grounded, whereas in the simulations we use Neumann zero boundary conditions, which cause the potential to change linearly at the sides (in the absence of space charge). This makes the horizontal guiding of streamers harder, but the simpler field configuration of the simulations allows for a more direct interpretation of guiding limits.

The simulations are performed on adaptive octree grids with local refinement. The refinement factor is always two, and we use the following refinement criterion:

- Refine if $\alpha \Delta x>1.0$, where $\alpha$ is the ionization coefficient and $\Delta x$ is the cell spacing

- Derefine if $\alpha \Delta x<0.1$ and $\Delta x<0.4 \mathrm{~mm}$

These criteria generate fine grids around streamer heads, on which their thin space charge layers are resolved. Furthermore, we ensure sufficient refinement around the initial conditions up to $10 \mathrm{~ns}$. In figure 7, an example of the charge density, numerical mesh and electric field is shown for a streamer developing in pure nitrogen.

The simulation code described above is fully open source and available through [32], and it will be described in more detail in an upcoming paper. The simulations presented in this article were performed on 16-core nodes and took 2-12 h, using up to $2 \times 10^{7}$ grid cells.

\section{Conditions for laser guiding}

In [13] we observed that guiding could occur in nitrogenoxygen mixtures with oxygen concentrations up to $0.5 \%$ (at 133 mbar, with our laser and voltage pulses). Below, we investigate the limits of guiding with simulations, experiments and plasma-chemical modelling, and we show that photoionization is the main factor limiting guiding in oxygen-rich mixtures.

\subsection{Simulations of guiding limits}

We have performed 3D simulations in a background field of $2 \mathrm{kV} / \mathrm{cm}$ at $133 \mathrm{mbar}$, using the following range of parameters:

- $\mathrm{N}_{2}$ with an $\mathrm{O}_{2}$ fraction of $0,0.1 \%$ or $1.0 \%$

- A preionized trail with $10^{8}, 10^{9}$ or $10^{10} \mathrm{~cm}^{-3}$ electrons and positive ions

Figure 8 shows a 3D view of the electron density at $t=146 \mathrm{~ns}$ for these nine cases; additional results at $t=166 \mathrm{~ns}$ are included for the case with the strongest laser ionization. Figure 9 shows the time evolution of the electric field and the electron density for the case with pure nitrogen and a $10^{9} \mathrm{~cm}^{-3}$ preionized trail. Note that the downwards propagating streamer branches, but that the symmetry in the initial conditions is preserved. This happens because there is no photoionization in pure nitrogen, which is the only source of random fluctuations in equation (1). Some of the other streamers in figure 8 also branch, but not in the direction perpendicular to the view.

In all simulations a background ionization level of $1.5 \times 10^{5} \mathrm{~cm}^{-3}$ was present, and the preionized trail was positioned as in figure 6. As already mentioned in section 2.3, we have used transport data for pure nitrogen for all mixtures, and thus excluded electron attachment / detachment reactions. In section 3.2, we show that these reactions cannot explain the lack of guiding in oxygen-rich mixtures. By using nitrogen transport data for all cases, we focus purely on the effects of photoionization.

3.1.1. Dependence on oxygen fraction. The guiding of streamers relies on a strong anisotropy in the electron density ahead of them, as illustrated in figure 10. In pure nitrogen this anisotropy is always present, because the preionized trail has a significantly higher degree of ionization than the background $\left(1.5 \times 10^{5} \mathrm{~cm}^{-3}\right)$. When oxygen is added to the mixture, photoionization will locally increase the 'background' ionization level around the discharge, and therefore inhibit guiding. This effect is illustrated in figure 11, which shows the electron density at $t=80 \mathrm{~ns}$ for the discharges of figures 8(f) and (j).

According to equation (6), the mean absorption length of ionizing photons is $l_{\mathrm{abs}} \approx 0.093 \mathrm{~mm} \cdot \mathrm{bar} / p_{\mathrm{O}_{2}}$, where $p_{\mathrm{O}_{2}}$ is partial pressure of oxygen. This gives $l_{\mathrm{abs}} \approx 70 \mathrm{~cm}$ for $\mathrm{N}_{2}$ with $0.1 \% \mathrm{O}_{2}$, and $l_{\mathrm{abs}} \approx 7 \mathrm{~cm}$ for the case with $1 \% \mathrm{O}_{2}$, explaining why there is significantly more photoionization present around the streamer in the latter case. 


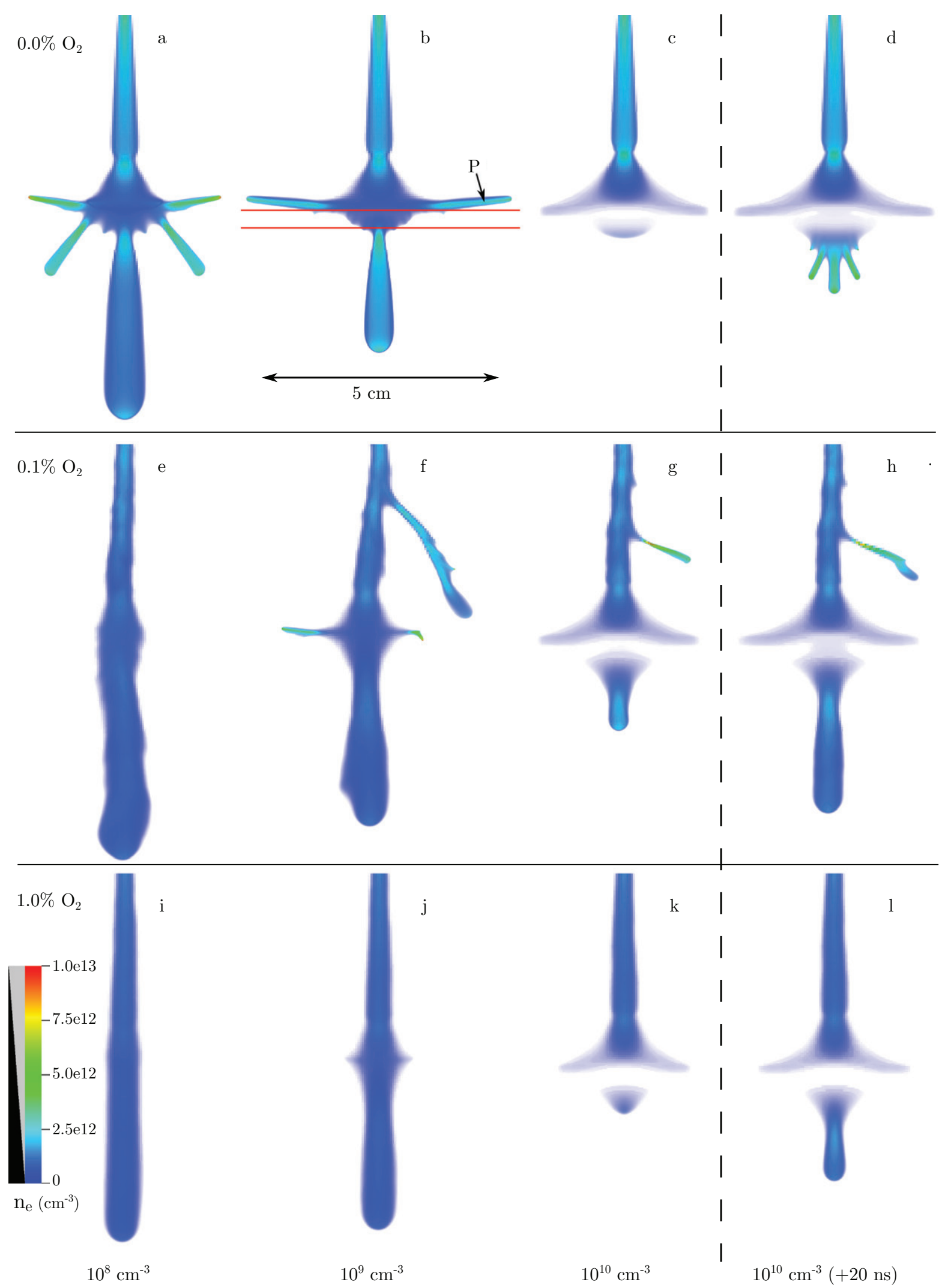

Figure 8. A $3 \mathrm{D}$ view of the electron density at $t=146 \mathrm{~ns}$ for simulations in a background field of $2 \mathrm{kV} \mathrm{cm}^{-1}$ at $133 \mathrm{mbar}$. The rows correspond to $\mathrm{N}_{2}$ with an $\mathrm{O}_{2}$ fraction of $0,0.1 \%$ and $1.0 \%$, and the columns to $10^{8}, 10^{9}$ and $10^{10} \mathrm{~cm}^{-3}$ preionized trails. For the $10^{10} \mathrm{~cm}^{-3}$ case, the right-most column shows additional results at $t=166 \mathrm{~ns}$. The pictures were generated with volume rendering in Visit [33], and the initial location of the preionized trail is outlined in red. The Monte Carlo photoionization breaks the symmetry present in the pure nitrogen cases. 


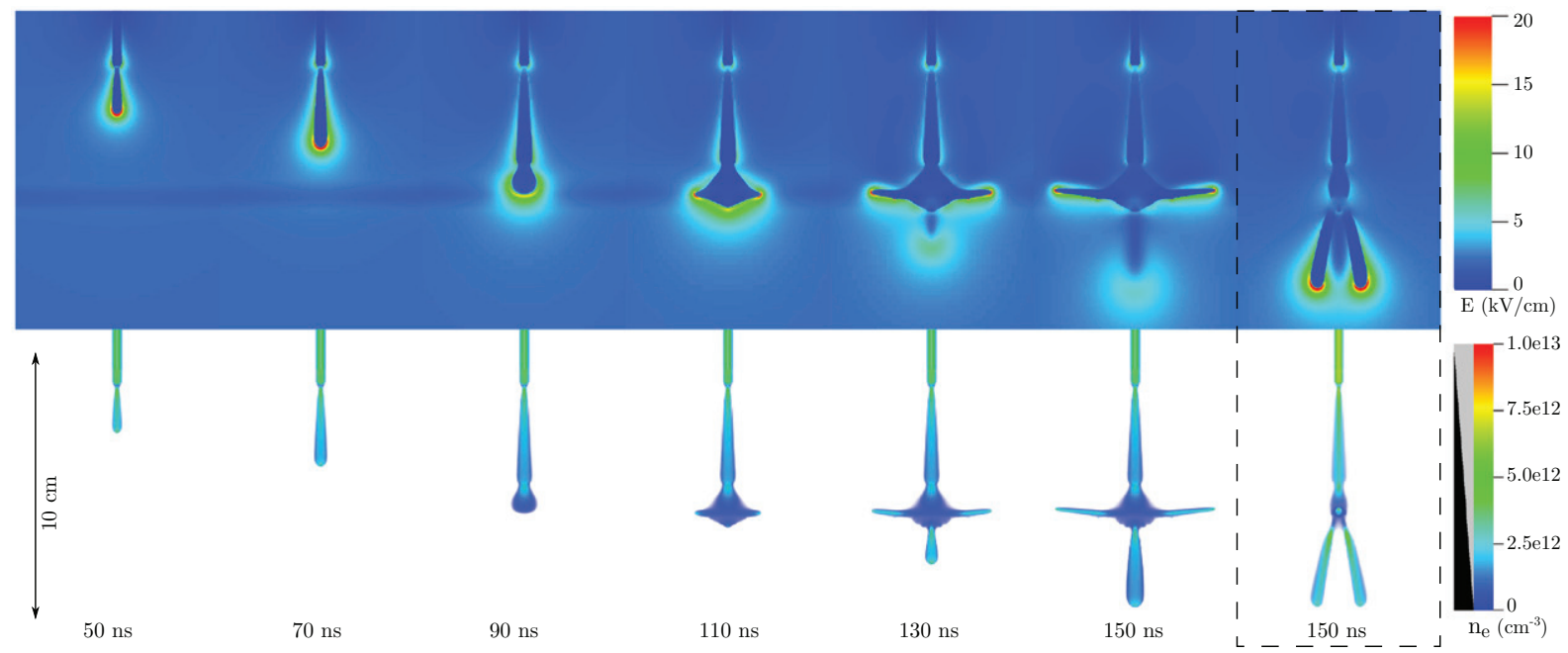

Figure 9. The time evolution of the discharge shown in figure $8(\mathrm{~b})$, propagating in pure nitrogen with a $10^{9} \mathrm{~cm}^{-3}$ preionized trail. Top row: cross sections of the electric field, bottom row: volume rendering of the electron density. For the rightmost figures, the viewpoint has been rotated by $90^{\circ}$, revealing that the downwards streamer has branched.

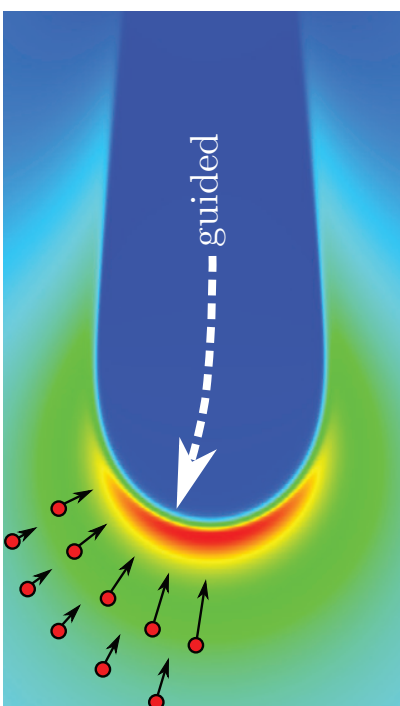

free

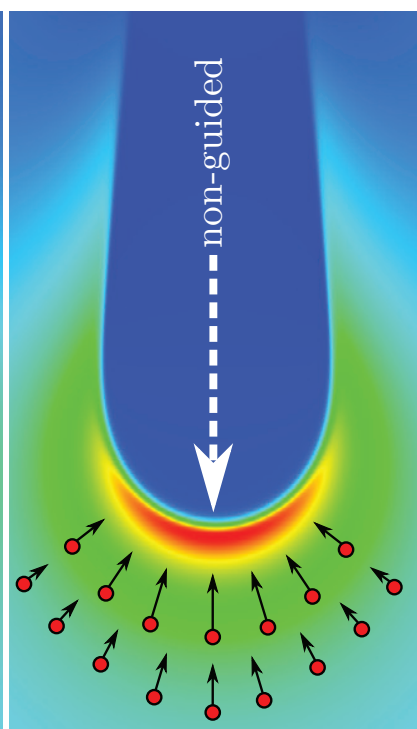

electrons

Figure 10. Schematic illustration of the guiding of positive streamers, with representative free electrons indicated as circles. The arrows indicate their drift velocity, and the background shows the electric field strength. If the density of free electrons is sufficiently anisotropic, the streamer will not grow in the direction of maximum field enhancement.

If in a given volume on average $N_{\gamma}$ photons are absorbed, then stochastic fluctuations have typical size $\sqrt{N_{\gamma}}$. Relative fluctuations are therefore of magnitude $1 / \sqrt{N_{\gamma}}$, becoming smaller at higher photoionization densities. The effect of these fluctuations can clearly be seen in figures 8 and 11: with $0.1 \%$ oxygen the streamer channel grows much more irregularly than with $1.0 \%$ oxygen. A similar observation was made in [34], though these calculations were performed in two dimensions, in which the interpretation of electron density

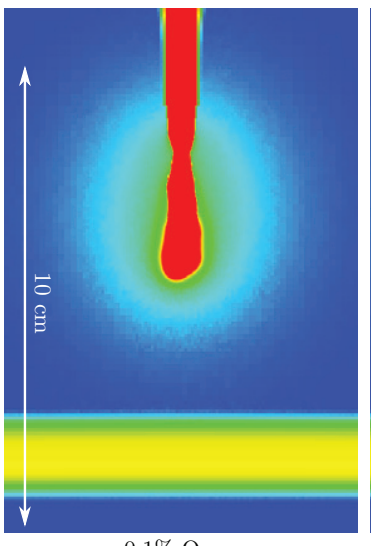

$0.1 \% \mathrm{O}_{2}$

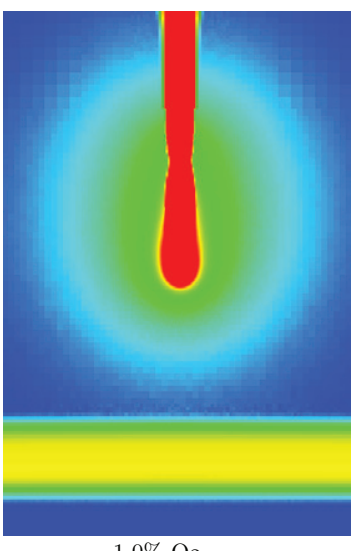

$1.0 \% \mathrm{O}_{2}$
Figure 11. Cross section through the simulation domain showing the electron density on a logarithmic scale at $t=50 \mathrm{~ns}$ for the discharges shown in figures $8(\mathrm{f})$ and $(\mathrm{j})\left(\mathrm{a} 10^{9} \mathrm{~cm}^{-3}\right.$ preionized trail, $0.1 \%$ and $1.0 \%$ oxygen). The electron density inside the streamer exceeds the scale; it is about $10^{13} \mathrm{~cm}^{-3}$, see figure 18 .

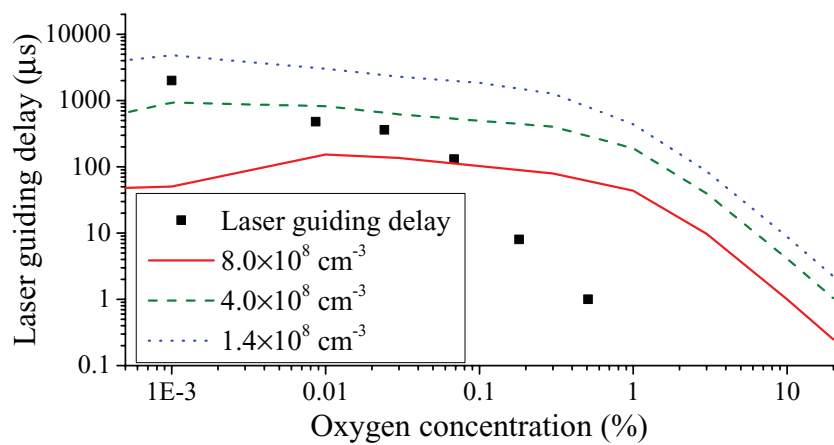

Figure 12. Symbols: maximum delay between laser and voltage pulse for which laser guiding is still observed as function of oxygen concentration in nitrogen. All at $133 \mathrm{mbar}, 6.7 \mathrm{kV}$ pulse amplitude and $600 \mathrm{~ns}$ pulse length. Lines: calculated time after laser pulse required to reach the indicated electron densities when starting from an initial electron density of $1 \cdot 10^{9} \mathrm{~cm}^{-3}$. 

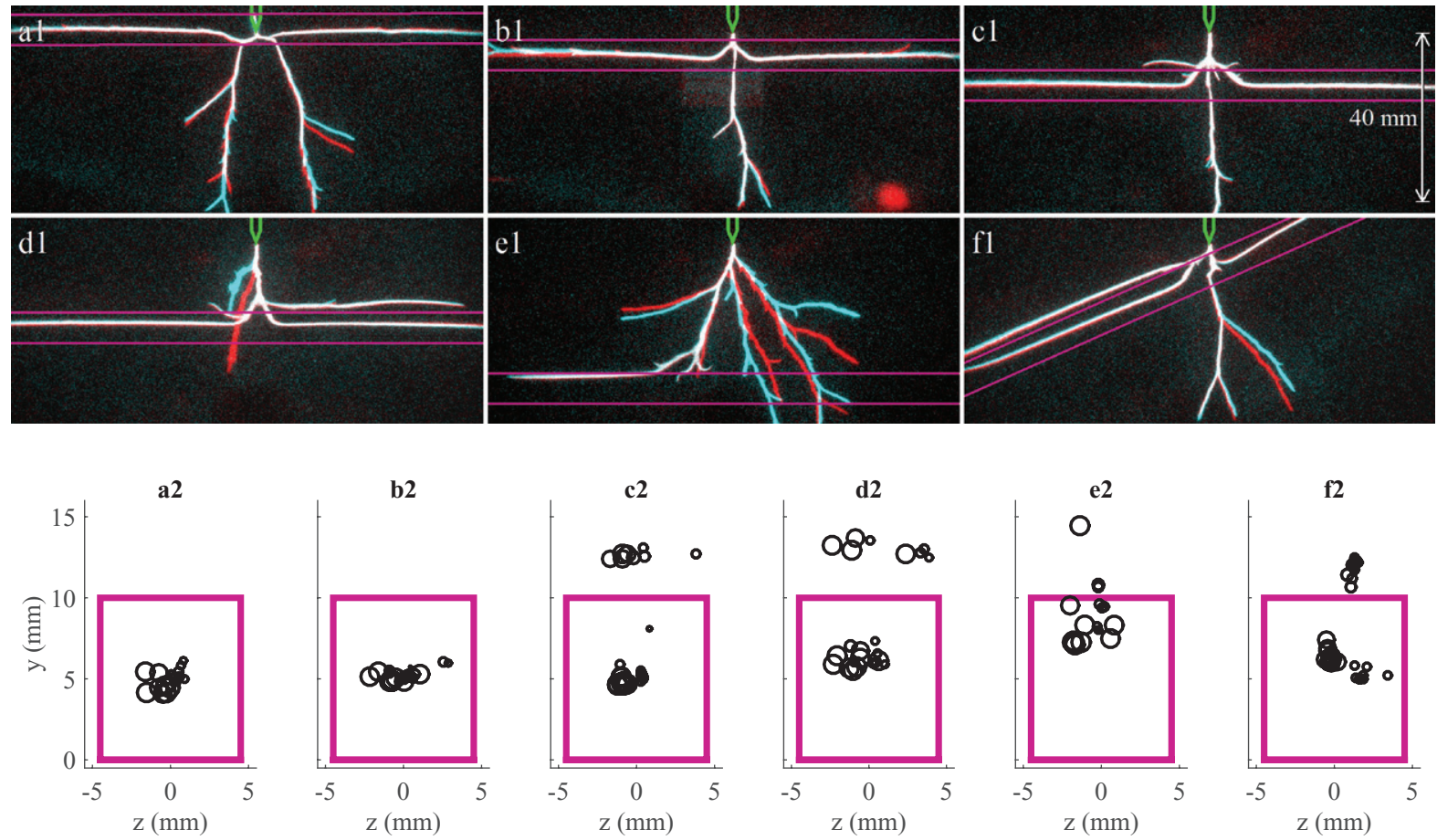

Figure 13. Stereoscopic images ((a1)-(f1)) and corresponding position plots ((a2)-(f2)) of laser-guided streamers for various laser positions. Measurements in 133 mbar pure nitrogen with a $5.9 \mathrm{kV}, 600 \mathrm{~ns}$ voltage pulse $1.1 \mu \mathrm{s}$ after the laser pulse. Each position plot contains points of about 10 discharge events. Images (a1)-(f1) were previously published in [13].

fluctuations due to single photons is not quite clear. The effect of electron density fluctuations on branching was also studied in three dimensions in [35], though these simulations were limited by computational constraints.

That photoionization inhibits guiding is most clearly seen for the $10^{8}$ and $10^{9} \mathrm{~cm}^{-3}$ preionized trails. Whereas guiding is observed in pure nitrogen, the streamers propagate almost straight down when $1.0 \%$ of oxygen is added. With $0.1 \%$ of oxygen the $10^{9} \mathrm{~cm}^{-3}$ trail still shows some guiding, whereas the $10^{8} \mathrm{~cm}^{-3}$ trail does not.

3.1.2. Dependence on preionization level in the trail. When the preionized trail is more highly ionized, streamer guiding is affected in multiple ways. First, guiding is enhanced, because the anisotropy in the electron density is increased. This is clearly visible when comparing figures 8(e), (f) and (g) or (i), (j) and (k). Second, higher ionization levels significantly slow down the streamer discharges, see figure 8 . This happens because the streamers grow wider and lose much of their field enhancement in the preionized trail. Third, due to the lower field enhancement the degree of ionization behind the streamer front is also locally reduced, in accordance with a general relation between maximal field strength and ionization degree in streamer channels [36]. For the $10^{10} \mathrm{~cm}^{-3}$ trail this effect is clearly visible in figure 8 (and was observed similarly in [37]: inside the trail, the degree of ionization is so low that our visualization becomes transparent. Fourth, more highly ionized trails have a higher conductivity, and therefore a stronger effect on the electric field. This effect is discussed in more detail in section 4 , see also figure 18 .

\subsection{Guiding experiments in nitrogen-oxygen mixtures}

In our laser-guiding experiments, we have determined the maximum time delay between laser and voltage pulse for which guiding is still observed. We define this delay such that roughly $50 \%$ of the discharges still show clear guiding. In figure 12 , the black squares indicate the maximum delay as a function of oxygen concentration. It is over $100 \mu$ s below $0.1 \%$ oxygen, but at higher concentrations it quickly decreases. Above $0.5 \%$ oxygen, no guiding was observed at all.

In a previous study with double voltage pulses [21], electron loss reactions could fully explain differences in the continuation of existing streamer paths. In figure 12, we have therefore drawn lines for the time it takes to reach a certain electron density due to such losses. These curves have been calculated using the ZDPlasKin code [38] with the same reactions and rates as described in [21]. Initially, a density of $1 \cdot 10^{9} \mathrm{~cm}^{-3}$ electrons and positive ions (divided over $\mathrm{O}_{2}^{+}$and $\mathrm{N}_{2}^{+}$through their concentration ratio) was present, at a pressure of 133 mbar and room temperature.

Figure 12 shows that the electron-loss times cannot account for the short laser guiding delay between $0.1 \%$ and $1 \%$ oxygen. We therefore conclude that guiding is prevented by a different oxygen-related mechanism: photoionization.

\section{Position of guided streamers}

The position of guided streamers is often slightly displaced with respect to the laser beam. Below we present experimental observations of this displacement for a variety of conditions, and we show that it is caused by electron drift. 

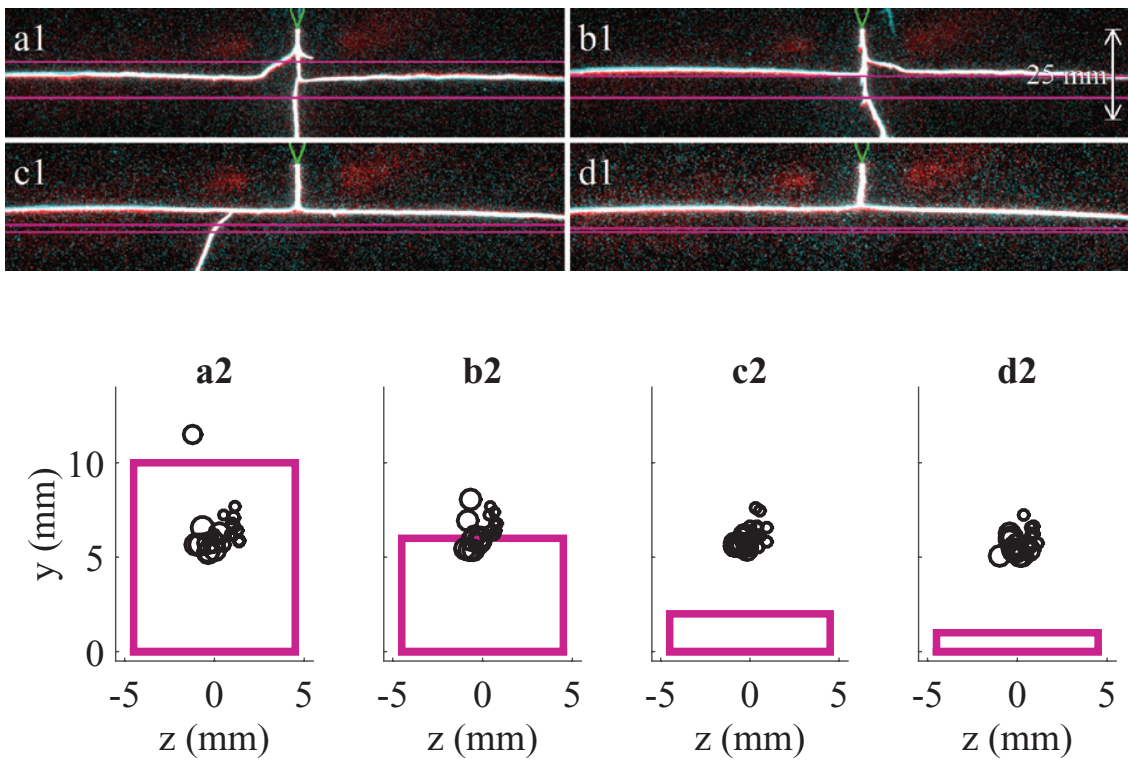

Figure 14. Stereoscopic images ((a1)-(d1)) and corresponding measured guiding positions ((a2)-(d2)) for different laser beam dimensions as indicated in (a2)-(d2). All data from measurements in pure nitrogen at 133 mbar with $4.8 \mathrm{kV}, 1000 \mathrm{~ns}$ long voltage pulses about $100 \mathrm{~ns}$ after the laser pulses.

Table 1. Overview of the effects of different parameter variations on the distance between the lowest guided streamers and the bottom of the laser beam.

\begin{tabular}{lcc}
\hline Parameter & Range & Effect \\
\hline $\begin{array}{l}\text { Delay between laser and voltage } \\
\text { pulse }\end{array}$ & $1-2500 \mu \mathrm{s}$ & $<1 \mathrm{~mm}$ \\
Laser energy (output power meter) & $8-15 \mathrm{mV}$ & $<1 \mathrm{~mm}$ \\
Laser beam height (figure 14) & $1-17 \mathrm{~mm}$ & $<1 \mathrm{~mm}$ \\
Pressure & $75-250 \mathrm{mbar}$ & $1 \mathrm{~mm}$ \\
$\begin{array}{l}\text { Distance bottom laser beam to } \\
\text { electrode tip (figure 13) }\end{array}$ & $3.5-52 \mathrm{~mm}$ & $3 \mathrm{~mm}$ \\
Voltage amplitude (figure 15) & $3.9-8.6 \mathrm{kV}$ & $5 \mathrm{~mm}$ \\
\hline
\end{tabular}

Note: The third column indicates the range over which this distance varies when the parameter is varied over the indicated range. Only one parameter is varied at the same time.

\subsection{Experimental observations}

Figures 13(a1)-(f1) show examples of guided streamers. These streamers propagate 5 to $15 \mathrm{~mm}$ above the bottom of the laser beam, as illustrated in in figures 13(a2)-(f2). (Here 'above' and 'bottom' are defined with respect to the picture's orientation.) Such an offset between the guided streamers and the laser beam bottom was present in all experimental observations, but seems counter-intuitive, because streamers are electrostatically attracted towards the bottom plate electrode. In some cases, there are channels that propagate above the entire laser-illuminated volume, as shown in figures 13(c2)-(e2) by the circles above the purple rectangle. Such guided streamers were only observed with another guided channel below them, as in figures 13(d1) and (f1).

We have studied the effect of various parameters on this position offset. Figure 14 shows that the distance between the lowest guided streamers and the bottom of the laser beam is independent of the height of the laser beam. For thin laser beams the guided streamers always propagate significantly above the area illuminated by the laser. The offset was independent of the width of the laser beam, and almost independent of pressure, laser energy and the laser-voltage pulse delay, as described in table 1 .

A parameter that significantly affects the position offset is the applied voltage, see figure 15. A higher applied voltage generally leads to more, thicker, and faster streamers [39, 40], and here it also leads to less guiding. However, streamers that are guided are located closer to the bottom of the laser beam. At the same pressure the location of the lowest guided streamer changes from $8.4 \mathrm{~mm}$ at $3.9 \mathrm{kV}$ to $3.3 \mathrm{~mm}$ at $8.6 \mathrm{kV}$.

\subsection{Estimate of electron drift}

The simplest explanation for the offset of the guided streamers with respect to the laser beam is electron drift in the background electric field before the streamer arrives. Here we estimate this drift distance for various conditions, using the electrostatic field calculations for the discharge vessel that were presented in figure 3 of [13] (scaled to the actual applied voltage).

First, the time it takes a streamer to reach a given location is estimated, which depends on the streamer's length and its average velocity. The streamer velocity was taken from a separate set of measurements of streamer velocity as function of applied voltage ${ }^{4}$. We assume that the streamer incepts at the start of the voltage pulse, and take its length equal to the sum of the horizontal and vertical distance to the electrode tip.

\footnotetext{
${ }^{4}$ This set of measurements was performed in another discharge vessel but with very similar general conditions (pressure, gas, point-plane distance). We used average velocities during the first few centimeters of the fastest streamers in a discharge. Due to small differences in voltage pulse shape and vessel geometry these measured velocities can deviate somewhat from the ones in the vessel used for laser guiding, but they still give a good indication of expected velocities. We fitted the measured velocities with a quadratic fit (see figure 16(c))
} 

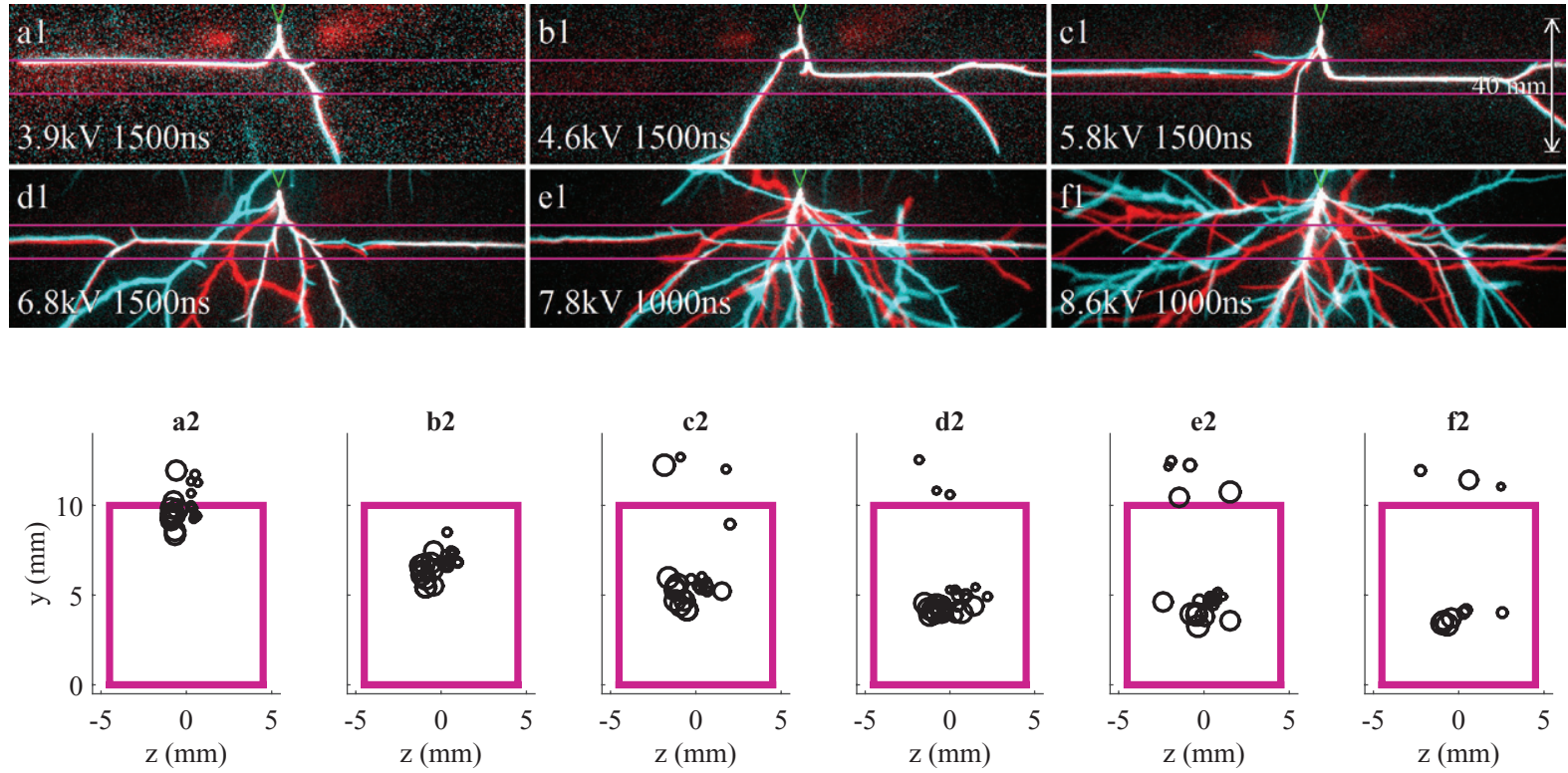

Figure 15. Stereoscopic images ((a)-(f)) and corresponding measured guiding positions ((g)-(1)) for different voltage pulses. Voltage amplitudes and pulse lengths are indicated in the stereoscopic images. All data from measurements in pure nitrogen with the voltage pulses about $100 \mathrm{~ns}$ after the laser pulses.
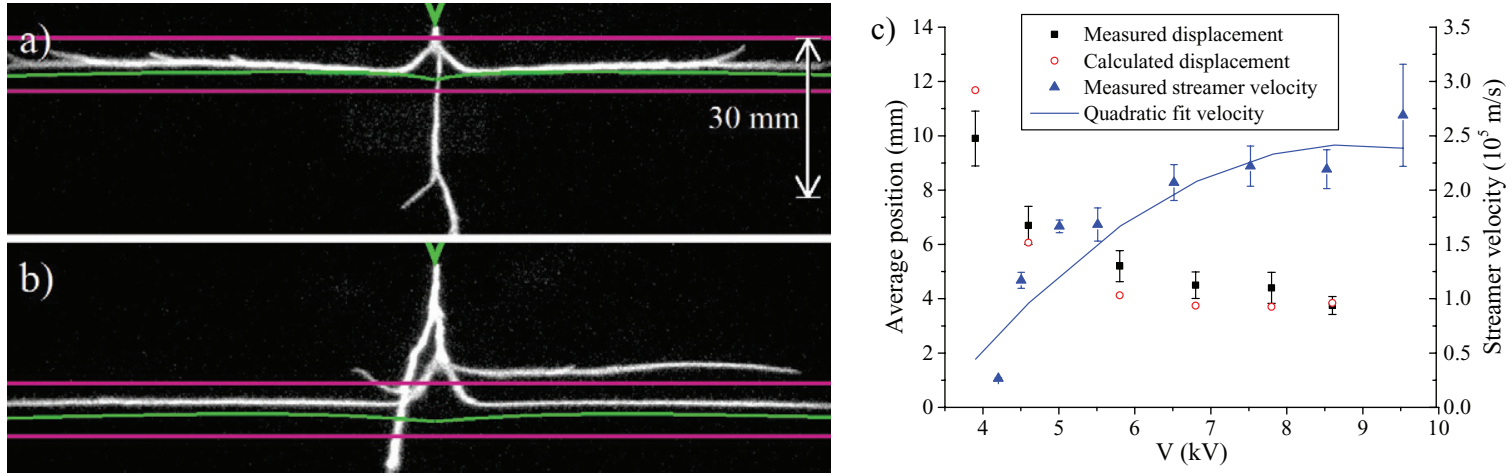

Figure 16. (a) and (b) streamer guiding images from figures 13(b) and (d). The green lines estimate where electrons from the bottom of the laser beam have drifted. (c) Measured average position (with standard deviation) of lowest guided streamers from the measurements of figure 15 as function of applied voltage together with estimated streamer velocities, a quadratic fit of these velocities and calculated vertical electron drift distances for these conditions.

This is a reasonable assumption for guided streamers towards most points below the tip (see e.g. figures 13(b)-(e)). Given a time $t$, the total drift distance of electrons at location $\vec{r}$ is then estimated as $-\mu_{\mathrm{e}} \vec{E}_{0}(\vec{r}) t$, where $\mu_{\mathrm{e}}$ is the field-dependent mobility and $\vec{E}_{0}$ the background field.

The procedure described above has been used to generate the green lines shown in figures 16(a) and (b), computed for the guided streamers from figures 13(b) and (d), respectively. These green lines represent the location of the electrons from the bottom of the laser (the lower purple line) at the estimated moment that the streamer arrives. Both the magnitude of the position offset (about $5 \mathrm{~mm}$ ) as well as its general shape show quite good agreement. Both in the image and in the calculated paths there is a maximum in the vertical offset at about $37 \mathrm{~mm}$ from the electrode axis. This can be explained as follows: the electrons closest to the electrode axis experience the highest field, but have less time before the streamer arrives and thus drift less far. The electrons far away from this axis do have

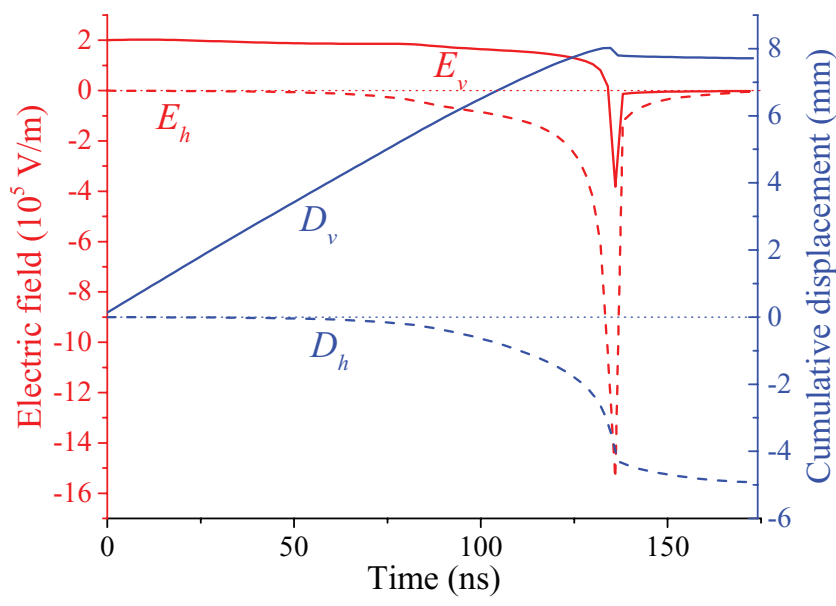

Figure 17. Horizontal ( $h$, dashed) and vertical ( $v$, solid) components of the electric field $E$ at position $P$ from the simulation shown in figure 8 (b) as function of time with calculated cumulative electron displacements $D$ due to these fields. 

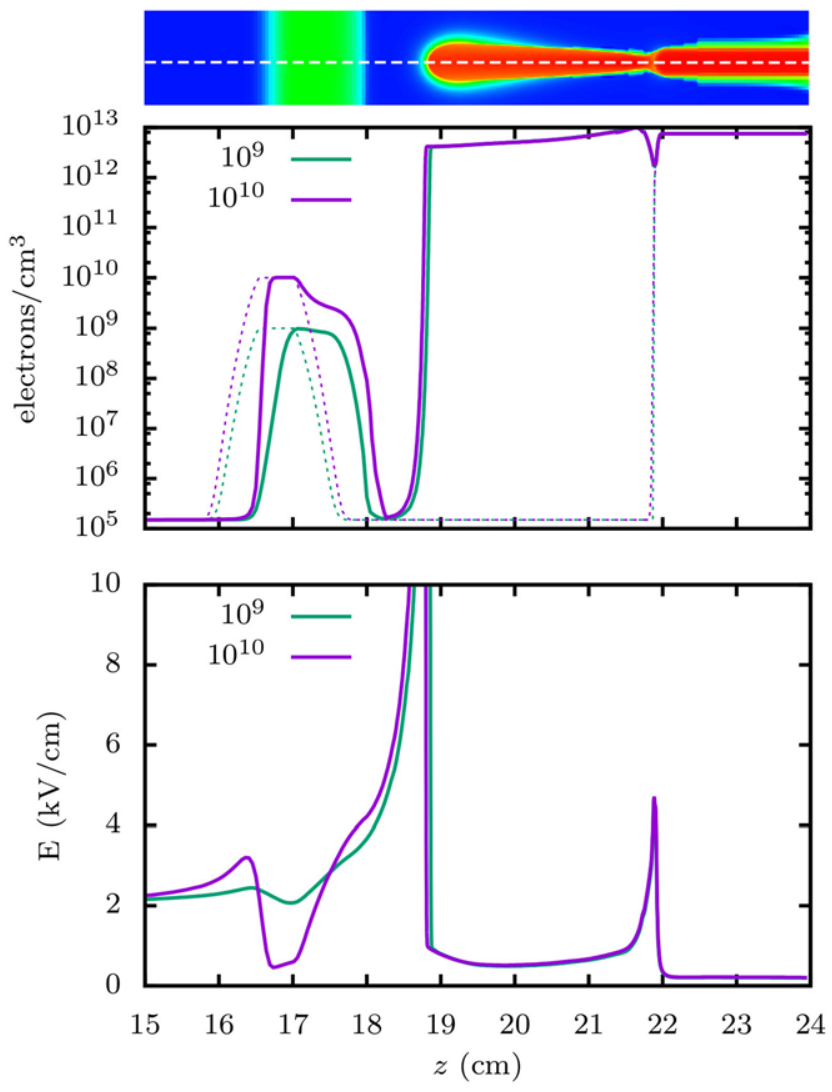

Figure 18. The on-axis electron density and electric field profile at $t=70 \mathrm{~ns}$, for the conditions of figures 8(b) and (c) (pure nitrogen, a $10^{9}$ and $10^{10} \mathrm{~cm}^{-3}$ preionized trail). Initial electron densities are shown as dotted lines. The top illustration shows an aligned cross section of the (logarithmic) electron density for the $10^{9}$ case.

more time to drift but there the field is much lower. Therefore, the electrons in between show the largest vertical drift.

In figure 16(c), the same procedure was performed for different applied voltages, to explain the observations of figure 15. The shown streamer positions are the average of the vertical positions of the streamers in the lowest groups in figures 15(a2)-(f2). The electron positions were calculated for electrons initially at the bottom of the laser beam, at a distance of $30 \mathrm{~mm}$ from the electrode axis. Again the measured results match the calculations quite closely, especially considering the uncertainties in the velocities (which in reality will vary over space and time) and the assumptions used in the calculations. Although the drift velocity increases with applied voltage, the faster increase in streamer velocity reduces the offset at higher voltages.

To check whether the vertical drift is indeed mostly due to the background field or also due the field of the approaching streamer, we have plotted the two non-zero components of the electric field from the simulation of figure 8(b) at position $P$ as function of time in figure 17. From these time-dependent fields we have derived the cumulative drift distance $D$ in both directions as function of time for an electron at this position by piecewise integration:

$$
D_{m}(t)=\int_{0}^{t} E_{m}(t) \mu_{e}(|\vec{E}(t)|) \mathrm{d} t
$$

for components $m=h, v$. ( $D$ is calculated at a fixed position, so the second-order effect of drift on the integrand is not included.)

The result for $D_{v}$ shows that although the electric field in the $z$-direction has its largest amplitude at the moment the streamer passes (around $t=135 \mathrm{~ns}$ ), most of the vertical drift occurs due to the background field, during the period before the streamer comes close. The horizontal drift is mostly due to the approaching streamer but this will not show in the vertical offset of the guided channel.

In the simulations from figure 8 , all guided streamers also propagate with an offset to the bottom of the original laser created channel due to the effect described above. However, they do not all propagate at the same offset and angle. In the simulations with a $10^{10} \mathrm{~cm}^{-3}$ preionized trail the offset is significantly smaller than for lower ionization levels. The reason is that such a high preionization density has an appreciable effect on the background electric field, as illustrated in figure 18. This figure shows the on-axis electron density and electric field at $t=70 \mathrm{~ns}$ for the simulations of figures 8(b) and (c) (pure nitrogen, a $10^{9}$ and $10^{10} \mathrm{~cm}^{-3}$ preionized trail). In the $10^{10} \mathrm{~cm}^{-3}$ case, the trail's interior is sufficiently conductive to screen the background electric field, which is therefore slightly enhanced on the outside. Most electrons are kept in place by the positive ions, which explains why the streamers guided by a $10^{10} \mathrm{~cm}^{-3}$ trail propagate almost horizontally in figure 8 .

It should be noted that in the simulation results like those from figure 8 the background field is fully vertical while in the experiments the point-plane geometry leads to a more complex field distribution. The more complex field was used in the calculations shown in figure 16 but not in those of figure 17.

In summary, the calculations above show that the offset of streamers can be fully explained by the drift of free electrons from the preionized trail, and that most of this drift takes place in the background electric field. This proves that the laser guiding itself is due to free electrons: if attached electrons or other heavy species would be responsible for laser guiding the expected offset would be much lower because the mobility of such heavier species is many orders of magnitude lower than that of electrons.

\section{Conclusions}

With measurements and 3D simulations we have shown that the guiding of streamers in nitrogen-oxygen mixtures is controlled by the distribution of free electrons. Experimental proof for this is the agreement between estimated electron drift distances and measured position offsets of guided streamers with respect to the laser beam. Our simulations confirm that most of the electron drift occurs in the background field, before a guided streamer comes close.

In the experiments, laser guiding was inhibited for oxygen concentrations above $0.5 \%$ in 133 mbar nitrogen, using an estimated laser preionization density of $10^{9} \mathrm{~cm}^{-3}$. In simulations with a preionized trail of the same density, guiding was still possible in nitrogen with $0.1 \%$ oxygen but not with $1 \%$ 
oxygen. With a ten times more strongly ionized trail, simulations showed that guided streamers lost most of their field enhancement. Furthermore, this level of preionization had significant space charge effects, preventing most of the electrons from drifting away. On the other hand, simulations with a $10^{8} \mathrm{~cm}^{-3}$ preionized trail showed no guiding with $0.1 \%$ oxygen. This seems to confirm that our experimental determination of the laser preionization density was quite accurate.

With plasma chemical modeling, we have shown that electron loss reactions cannot explain the lack of guiding at higher oxygen fractions at 133 mbar. We therefore conclude that laser guiding is predominantly inhibited by photoionization, as also demonstrated in our simulations. We remark that at higher pressures, electron loss processes become more important because they often scale quadratically with density [13].

\section{Acknowledgments}

SN was supported by FY2012 Researcher Exchange Program between the Japan Society for the Promotion of Science JSPS and The Netherlands' Organisation for Scientific Research NWO, JT by STW-project 10755 and ET by JSPS KAKENHI Grant Number 24560249.

\section{References}

[1] Luque A, Ratushnaya V and Ebert U 2008 Positive and negative streamers in ambient air: modeling evolution and velocities J. Phys. D: Appl. Phys. 41234005

[2] Ebert U and Sentman D D 2008 Streamers, sprites, leaders, lightning: from micro- to macroscales J. Phys. D: Appl. Phys. 4123030

[3] Nijdam S, Bruggeman P, van Veldhuizen E M and Ebert U 2012 Chapter. An introduction to nonequilibrium plasmas at atmospheric pressure Plasma Chemistry and Catalysis in Gases and Liquids (Weinheim: Wiley)

[4] van Veldhuizen E M (ed) 2000 Electrical Discharges for Environmental Purposes: Fundamentals, Applications (New York: Nova Science Publishers)

[5] Fridman G, Friedman G, Gutsol A, Shekhter A B, Vasilets V N and Fridman A 2008 Applied plasma medicine Plasma Process. Polym. 5 503-33

[6] Starikovskii A Y, Anikin N B, Kosarev I N, Mintoussov E I, Nudnova M M, Rakitin A E, Roupassov D V, Starikovskaia S M and Zhukov V P 2008 Nanosecondpulsed discharges for plasma-assisted combustion and aerodynamics J. Propulsion Power 241182

[7] Pasko V P 2006 Chapter. Theoretical modeling of sprites and jets Sprites, Elves and Intense Lightning Discharges (NATO Science Series II: Mathematics, Physics, Chemistry vol 225) (New York: Springer) pp 253-311

[8] Ebert U, Nijdam S, Li C, Luque A, Briels T and van Veldhuizen E 2010 Review of recent results on streamer discharges and discussion of their relevance for sprites and lightning J. Geophys. Res._Space Phys. 115 A00E43

[9] Bonaventura Z, Bourdon A, Celestin S and Pasko V P 2011 Electric field determination in streamer discharges in air at atmospheric pressure Plasma Sources Sci. Technol. 20035012

[10] Nijdam S, van de Wetering F M J H, Blanc R, van Veldhuizen E M and Ebert U 2010 Probing photoionization: experiments on positive streamers in pure gases and mixtures J. Phys. D: Appl. Phys. 43145204
[11] Wladimir An, Baumung K and Bluhm H 2007 Underwater streamer propagation analyzed from detailed measurements of pressure release J. Appl. Phys. 101053302

[12] Nijdam S, Geurts C G C, van Veldhuizen E M and Ebert U 2009 Reconnection and merging of positive streamers in air J. Phys. D: Appl. Phys. $\mathbf{4 2} 045201$

[13] Nijdam S, Takahashi E, Teunissen J and Ebert U 2014 Streamer discharges can move perpendicularly to the electric field New J. Phys. 16103038

[14] Nijdam S and Takahashi E 2015 Guiding of positive streamers in nitrogen, argon and $\mathrm{N}_{2}-\mathrm{O}_{2}$ mixtures by very low $n_{e}$ laserinduced pre-ionization trails 32nd Int. Conf. of Phenomena in Ionized Gases (Iaşi, Romania, 26-31 July 2015)

[15] Koopman D W and Wilkerson T D 1971 Channeling of an ionizing electrical streamer by a laser beam J. Appl. Phys. 42 1883-6

[16] Fujii T, Miki M, Goto N, Zhidkov A, Fukuchi T, Oishi Y and Nemoto K 2008 Leader effects on femtosecond-laserfilament-triggered discharges Phys. Plasmas 15013107

[17] Leonov S B, Firsov A A, Shurupov M A, Michael J B, Shneider M N, Miles R B and Popov N A 2012 Femtosecond laser guiding of a high-voltage discharge and the restoration of dielectric strength in air and nitrogen Phys. Plasmas 19123502

[18] Clerici M et al 2015 Laser-assisted guiding of electric discharges around objects Sci. Adv. 1 e1400111

[19] Liu N and Pasko V P 2004 Effects of photoionization on propagation and branching of positive and negative streamers in sprites J. Geophys. Res. 1091

[20] Nijdam S, Wormeester G, van Veldhuizen E M and Ebert U 2011 Probing background ionization: positive streamers with varying pulse repetition rate and with a radioactive admixture J. Phys. D: Appl. Phys. 44455201

[21] Nijdam S, Takahashi E, Markosyan A and Ebert U 2014 Investigation of positive streamers by double pulse experiments, effects of repetition rate and gas mixture Plasma Sources Sci. Technol. 23025008

[22] Nijdam S, Miermans K, van Veldhuizen E M and Ebert U 2011 A peculiar streamer morphology created by a complex voltage pulse IEEE Trans. Plasma. Sci. 39 2216-7

[23] Pancheshnyi S 2015 Photoionization produced by low-current discharges in $\mathrm{O}_{2}$, air, $\mathrm{N}_{2}$ and $\mathrm{CO}_{2}$ Plasma Sources Sci. Technol. 24015023

[24] Wormeester G, Pancheshnyi S, Luque A, Nijdam S and Ebert U 2010 Probing photo-ionization: simulations of positive streamers in varying $\mathrm{N}_{2}: \mathrm{O}_{2}$-mixtures $J$. Phys. $D$ : Appl. Phys. $\mathbf{4 3} 505201$

[25] Pancheshnyi S 2005 Role of electronegative gas admixtures in streamer start, propagation and branching phenomena Plasma Sources Sci. Technol. 14645

[26] Nijdam S, Moerman J S, Briels T M P, van Veldhuizen E M and Ebert U 2008 Stereo-photography of streamers in air Appl. Phys. Lett. 92101502

[27] Teunissen J 2015 3D simulations, analysis of pulsed discharges PhD Thesis Technische Universiteit Eindhoven http://repository.tue.nl/801516

[28] Multiple databases www.lxcat.net retrieved on February 26 2015

[29] Hagelaar G J M and Pitchford L C 2005 Solving the Boltzmann equation to obtain electron transport coefficients and rate coefficients for fluid models Plasma Sources Sci. Technol. 14722

[30] Zhelezniak M B, Mnatsakanian A K H and Sizykh S V 1982 Photoionisation of nitrogen and oxygen mixtures by radiation from a gas discharge High Temp. 20357

[31] Chanrion O and Neubert Jul T 2008 A PIC-MCC code for simulation of streamer propagation in air J. Comput. Phys. 227 7222-45 
[32] Multiscale Dynamics group CWI multiscale dynamics webpage http://cwimd.nl

[33] Childs $\mathrm{H}$ et al 2012 VisIt: an end-user tool for visualizing, analyzing very large data High Performance VisualizationEnabling Extreme-Scale Scientific Insight (London: Chapman and Hall) pp 357-72

[34] Xiong Z and Kushner M J 2014 Branching and path-deviation of positive streamers resulting from statistical photon transport Plasma Sources Sci. Technol. 23065041

[35] Luque A and Ebert U 2011 Electron density fluctuations accelerate the branching of streamer discharges in air Phys. Rev. E 84046411

[36] Li C, Brok W J M, Ebert U and van der Mullen J J A M 2007 Deviations from the local field approximation in negative streamer heads J. Appl. Phys. 101123305
[37] Luque A and Gordillo-Vázquez F J 2011 Sprite beads originating from inhomogeneities in the mesospheric electron density Geophys. Res. Lett. 38 L04808

[38] Pancheshnyi S, Eismann B, Hagelaar G J M and Pitchford L C 2008 Computer code ZDPlasKin (University of Toulouse, LAPLACE, CNRS-UPS-INP, Toulouse, France)

[39] Briels T M P, Kos J, Winands G J J, van Veldhuizen E M and Ebert U 2008 Positive and negative streamers in ambient air: measuring diameter, velocity and dissipated energy J. Phys. D: Appl. Phys. 41234004

[40] Briels T M P, van Veldhuizen E M and Ebert U 2008 Positive streamers in air and nitrogen of varying density: experiments on similarity laws J. Phys. D: Appl. Phys. 41234008 